\title{
A Model for Capturing and Tracing Architectural Designs
}

\author{
M. Luciana Roldán, Silvio Gonnet, Horacio Leone \\ CIDISI, Universidad Tecnológica Nacional \\ INGAR, Universidad Tecnológica Nacional, CONICET \\ Avellaneda 3657, 3000, Santa Fe, Argentina \\ \{1roldan, sgonnet, hleone\}@ceride.gov.ar
}

\begin{abstract}
Software architecture constitutes the primary design of a software system. Consequently, architectural design decisions involved in architecture design have a key impact on the system in such aspects as future maintenance costs, resulting quality, and timeliness. However, the applied knowledge employed and the design decisions taken by software architects are not explicitly represented in the design despite their important role; consequently, they remain in the mind of designers and are lost with time. In this work, a model for capturing and tracing the products and architectural design decisions involved in software architecture design processes is proposed. An operational perspective is considered in which design decisions can be modelled by means of design operations. The basic ontology of situation calculus is adopted to formally model the evolution of a software architecture.
\end{abstract}

\section{Introduction}

Software Architecture Design Process (SADP) involves several activities such as exploration, evaluation and composition of design alternatives which make it a difficult, complex process [1]. In order to address those activities, the research community has been working intensively in the achievement of modelling languages $[2,3]$, design methods [4] and computer environments for architect assistance [1,5]. Those tools are basically focused on assisting designers in generating a software architecture design to satisfy a set of requirements. However, documentation of associated rationale, design decisions, and applied knowledge are often omitted. Such omissions stem from the fact that such information may be intuitive or obvious to the architects involved in the design process, or from the lack of adequate computer-aided environments that allow support design processes. Thus, most 
architectural design knowledge and architectural design decisions taken through SADP remain in the minds of experienced designers, and are lost with time. Consequently, capturing design decisions is of great importance to capitalize previous designs and to provide the foundations for learning and training activities. Precisely, this latter issue has been the goal of other contributions [6,7] which recognise that the design rationale should be incorporated into the SADP.

Therefore, this work introduces a model for capturing and tracing the SADP and its products. Its goals are to make explicit the states of the SADP and the way in which they were generated. The model is based on a generic Process Version Administration Model (PVAM) [8], which provides mechanisms for capturing and managing versions generated during the course of an engineering design project.

In the next section a conceptual model is presented, introducing the extensions for making PVAM applicable to SADP. After that, the operation capturing system is described, where the products and operations of the SADP are represented. The proposed model is illustrated in Section 4 with a case study about the design of a monitoring system for an industrial process. Finally, conclusions and future research guidelines are discussed.

\section{A Conceptual Model for Capturing Architectural Design Processes}

The proposed scheme considers the SADP as a sequence of activities operating on the products of the design process, which are called design objects. Examples of design objects are components and connectors of the architecture being designed, or functional and quality requirements and scenarios to be met. Naturally, these objects evolve as the SADP takes place, giving rise to several versions. In order to maintain these versions, the previously proposed PVAM [8] is considered. The general scheme employed in such approach represents a design object at two levels, the repository and the versions level. Each model version is generated from views of a repository that keeps all the objects that have been created and modified due to the model evolution during a design project. The elements constituting the repository are called versionable objects. A versionable object represents the artifact that can evolve during a design project, whose history is desirable to be kept during the modelling process. Furthermore, relationships among the different objects are maintained in the repository.

At the versions level, the evolution of versionable objects contained in the repository is explicitly represented. A model version consists of a set of instances of object versions which represent the versions of the objects that compose a given model at a time point. The relationships between a versionable object and one of its object versions is represented by the version $(v, o)$ predicate. Therefore, a given versionable object keeps a unique instance in the repository and the versions it assumes in different model versions belong to the versions level.

Based on that scheme, the model evolution is posed as a history made up of discrete situations. The situation calculus [9] is adopted for modelling such version generation process. A new model version $m_{n}$ is generated when an activity $a$ is 
executed. An activity $a$ is materialised by a sequence of operations $\phi$ and the new model version $m_{n}$ is the result of applying such sequence $\phi$ to the components of a previous model version $m_{p}$. In the context given by the design process, it is possible to assimilate each new generated model version with a situation and each action with a sequence of operations which is applied on a precedent model version. Therefore, the new model version $m_{n}$ is achieved by performing the following evaluation: $\operatorname{apply}\left(\phi, m_{p}\right)=m_{n}$.

The primitive operations that were proposed to represent the transformation of model versions are add, delete, and modify. By using the add(v) operation, an object version that did not exist in a previous model version can be incorporated into a successor model version. Conversely, the delete(v) operation eliminates an object version that existed in the previous model version. Also, if a design object has a version $v_{p}$, the modify $\left(v_{p}, v_{s}\right)$ operation creates a new version $v_{s}$ of the existing design object, where $v_{s}$ is a successor version of $v_{p}$. Thus, an object version $v$ is added after applying the sequence of operations $\phi$ to model version $m$ when the new version $v$ is created by means of an add or modify operation (Expression 1). On the other hand, the Expression 2 represents the fact that an object version $v$ is deleted after applying the sequence of operations $\phi$ to model version $m$ when the version $v$ is deleted by the delete or modify operation.

$$
(\forall \phi, v, m) \operatorname{add}(v) \in \phi \vee\left(\exists v_{p}\right) \operatorname{modify}\left(v_{p}, v\right) \in \phi \Rightarrow \operatorname{added}(v, \operatorname{apply}(\phi, m))
$$

$$
(\forall \phi, v, m) \operatorname{delete}(v) \in \phi \vee\left(\exists v_{s}\right) \operatorname{modify}\left(v, v_{s}\right) \in \phi \Rightarrow \operatorname{deleted}(v, \operatorname{apply}(\phi, m))
$$

From these definitions, and using the format of successor state axioms proposed by [9], a formal specification of the cases in which an object version belongs to a model version is presented. In Expression 3, the predicate belong $(v, m)$ is true when object version $v$ belongs to model version $m$. Thus, an object version $v$ belongs to a model version that arises after applying the sequence of operations $\phi$ to model version $m$, if and only if one of the following conditions is met: (i) $v$ is added when the new version is created $(\operatorname{added}(v, \operatorname{apply}(\phi, m)))$; or (ii) $v$ already belonged to the previous model version $m(\operatorname{belong}(v, m))$ and it is not deleted when $\phi$ is applied to it $(\neg \operatorname{deleted}(v, \operatorname{apply}(\phi, m)))$.

$$
\begin{gathered}
(\forall \phi, v, m) \text { belong }(v, \operatorname{apply}(\phi, m)) \Leftrightarrow \\
(\operatorname{belong}(v, m) \vee \operatorname{added}(v, \operatorname{apply}(\phi, m))) \wedge(\neg \operatorname{deleted}(v, \operatorname{apply}(\phi, m)))
\end{gathered}
$$

From this expression, the object versions belonging to a model version can be determined. Then, it is possible to reconstruct a model version $m_{i+1}$ by applying all operation sequences from the initial model version $m_{0}$.

Once the versions belonging to a model version are defined, the relationships existing among object versions have to be specified. First, it should be noted that in this proposal, object versions belonging to a model version are not explicitly associated to other versions belonging to the same model version. These links are represented at the repository level. Consequently, the relationship existing between two object versions must be inferred from the relationship established between the versionable objects that have been versioned by them. This fact is represented in 
Expression 4, in which an association $a_{k}$ is inferred between two object versions $v_{1}$ and $v_{2}$ belonging to the same model version $m$ (inferredAssociation $\left(a_{k}, v_{1}, v_{2}, m\right)$ ), if and only if there exists an association $a_{k}$ between the two versionable objects $o_{1}$ and $o_{2}\left(\operatorname{association}\left(a_{k}, o_{1}, o_{2}\right)\right.$ ), of which $v_{1}$ and $v_{2}$ are versions, respectively (version $\left(v_{l}\right.$, $\left.o_{1}\right)$ and version $\left.\left(v_{2}, o_{2}\right)\right)$.

$$
\begin{gathered}
\left(\forall v_{l}, v_{2}, m, a_{k}\right) \text { inferredAssociation }\left(a_{k}, v_{l}, v_{2}, m\right) \Leftrightarrow \\
\left(\exists o_{1}, o_{2}\right) \text { belong }\left(v_{1}, m\right) \wedge \operatorname{belong}\left(v_{2}, m\right) \wedge \operatorname{version}\left(v_{l}, o_{1}\right) \wedge \operatorname{version}\left(v_{2}, o_{2}\right) \wedge \\
\text { association }\left(a_{k}, o_{1}, o_{2}\right)
\end{gathered}
$$

The primitive operations add, delete, and modify introduced are not enough to capture and trace a SADP execution. Then, PVAM must be extended in terms of the suitable operations for this design domain, like the ones listed in Table 1. This operations range from the most basic to the most complex ones:

- Basic: operations that allow creating and deleting basic design objects (like components and connectors);

- Special: more complex operations that involve object refinement or delegation;

- Styles/Mechanisms application: these operations generate a new set of design objects which have a configuration based on an architectural style; or even if they

\begin{tabular}{|c|c|c|}
\hline \multicolumn{3}{|c|}{ Basic Operations } \\
\hline $\begin{array}{l}\text { addComponent } \\
\text { addConnector } \\
\text { addFunctionalRequirement } \\
\text { addPort } \\
\text { addProperty } \\
\text { addQualityRequirement } \\
\text { addResponsibility } \\
\text { addRole }\end{array}$ & $\begin{array}{l}\text { addScenario } \\
\text { addTypeComponent } \\
\text { addTypeConnector } \\
\text { deleteComponent } \\
\text { deleteConnector } \\
\text { deleteFunctionalRequirement } \\
\text { deletePort } \\
\text { deleteProperty }\end{array}$ & $\begin{array}{l}\text { deleteQualityRequirement } \\
\text { deleteResponsibility } \\
\text { deleteRole } \\
\text { deleteScenario } \\
\text { deleteTypeComponent } \\
\text { deleteTypeConnector }\end{array}$ \\
\hline \multicolumn{3}{|c|}{ Special Operations } \\
\hline $\begin{array}{l}\text { refineComponent } \\
\text { refineResponsibility }\end{array}$ & $\begin{array}{l}\text { delegateResponsibility } \\
\text { delegateScenario }\end{array}$ & verifyScenario \\
\hline \multicolumn{3}{|c|}{ Styles/Mechanisms application } \\
\hline $\begin{array}{l}\text { applyIntermediaryBlackboard } \\
\text { applyControlLoop }\end{array}$ & $\begin{array}{l}\text { applyRuleEngine } \\
\text { applyClientServer }\end{array}$ & applyPoolOfConnections \\
\hline
\end{tabular}
do not modify the model structure, they affect certain design objects properties.

Table 1. Possible Operations for the Software Architecture Design Domain

These operations are defined in terms of primitive operations as add(c), and nonprimitive ones (see Table 1), as addPort $(c, p)$. The execution of one of these operations implies that a sequence of primitive operations add, delete, and/or modify are applied to a previous model version, which results in a new model version. From this, it is possible to express these operations in terms of added and deleted predicates introduced in Expressions 1 and 2. For illustration purposes, let us consider the addComponent $\left(s, c, l_{\text {Resps }}, l_{\text {Ports }}\right)$ operation. It adds a component $c$ to a system $s$. Therefore, if it is applied to a model version $m$, then a version of a 
component $c$ having a set of responsibilities $r$ and ports $p$, will belong to the successor model version $(\operatorname{apply}(\phi, m))$, as it is defined in Expression 5 .

$$
\begin{gathered}
\left(\forall \phi, s, c, l_{\text {Resps }}, l_{\text {Ports }}, m\right) \text { addComponent }\left(s, c, l_{\text {Resps }}, l_{\text {Ports }}\right) \in \phi \Rightarrow \\
\text { added }(c, \operatorname{apply}(\phi, m)) \wedge \operatorname{added}(\operatorname{rel}(s, c), \operatorname{apply}(\phi, m)) \wedge \\
\left(\left(\forall r \in l_{\text {Resps }}\right) \operatorname{added}(r, \operatorname{apply}(\phi, m)) \wedge \operatorname{added}(\operatorname{rel}(c, r), \operatorname{apply}(\phi, m))\right) \wedge \\
\left(\left(\forall p \in l_{\text {Ports }}\right) \operatorname{added}(p, \operatorname{apply}(\phi, m)) \wedge \operatorname{added}(\operatorname{rel}(c, p), \operatorname{apply}(\phi, m))\right)
\end{gathered}
$$

Similarly to Expression 5, the definition of new operations allows enlarging the set of operations. This can be done without modifying the successor state axiom (Expression 3).

The precondition for applying the addComponent operation is specified in Expression 6, where the poss $(o p, m)$ predicate expresses that an operation op is applicable to a given model version $m$.

$$
\begin{gathered}
\left(\forall s, c, l_{\text {Resps }}, l_{\text {Ports }}, m\right) \text { poss }\left(\text { addComponent }\left(s, c, l_{\text {Resp }}, l_{\text {Ports }}\right), m\right) \Leftrightarrow \\
\text { belong }(s, m) \wedge \neg \operatorname{belong}(c, m) \wedge \\
\left(\forall r \in l_{\text {Resps }}\right) \neg \operatorname{belong}(r, m) \wedge\left(\forall p \in l_{\text {Ports }}\right) \neg \operatorname{belong}(p, m)
\end{gathered}
$$

\section{The Version Support System for Capturing Architectural Design Processes}

\subsection{Defining the Operations Model}

The class diagram illustrated in Fig. 1 shows the main concepts of PVAM introduced in the previous section. The relationship between a versionable object and one of its object versions is represented by the version relationship. Furthermore, it is assumed that design objects are identified and classified according to the different types (see Section 3.2). The design object type is represented by ModellingConcept class (Fig. 1). 


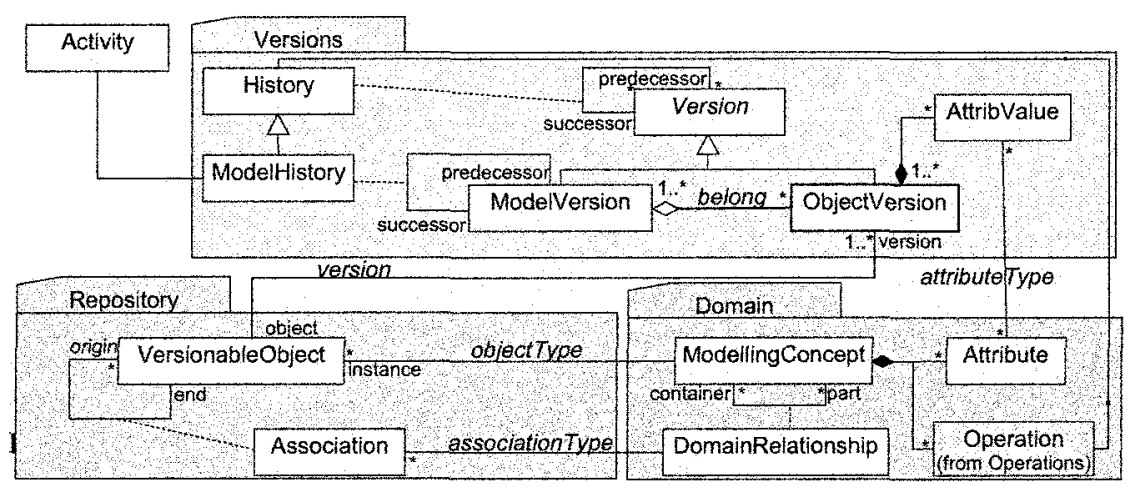

As outlined before, each transformation operation applied to a model version incorporates the necessary information to trace a model evolution. This information is represented by history relationships between the object versions to which the operation is applied and the ones arising as the result of its execution (Fig. 1). In order to represent architecture evolution, a model version has zero or more successor model versions (noted by * cardinality at successor role of History association shown in Fig. 1).

PVAM must be capable of extending in terms of the suitable operations for SADP domain. Subsequently, in this section the operation model is presented, which allows specifying and instantiating specific domain operations.

Operations are associated with a modelling concept and are defined as ordered sets of commands (Fig. 2). Those commands can be primitives or operations that can be used to define other operations. Primitives encapsulate the semantics defined by Expressions 1, 2 and 3. The execution of an operation generates one or more results, which can be a set of versions. Furthermore, history class is instantiated, linking the predecessor with the successor versions. 


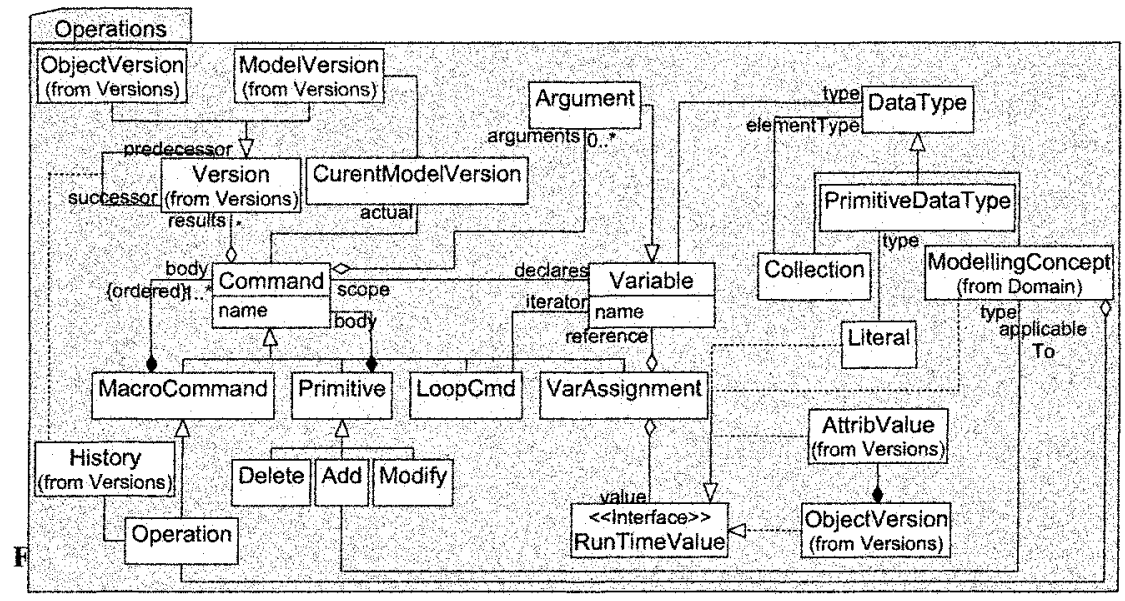

The operation definition is represented using the basic structure of the Abstract Syntax Kernel Metamodel for Expressions defined by the UML 2.0 OCL Specification [10]. To implement operations, the well-known Command design pattern was used [11]. Therefore, a command abstract class is introduced into the Operations package illustrated in Fig. 2. An operation is defined as a macro command (MacroCommand class), a subclass of command that simply executes a sequence of commands. Therefore, when an operation is specified, it is necessary to define both the arguments and the body of the operation. The commands that constitute its body are some other already defined commands, which are available for use in the specification (primitives, loop, variable assignment, or other operations). Note that the modelling concept over which an operation is applied must be explicitly indicated. Furthermore, there are other concrete classes that specialise the command class, and that can be part of a macro command. One of them is the LoopCmd, which represents a loop construct over a collection variable and has a body that is executed for each element in the collection. Another valid command is VariableAssignment that represents the assignment of a value to a variable of a given type.

As shown in Fig. 2, every command has one or more data typed arguments. Arguments are considered as a kind of variable. A variable can be also declared and used in the body of an operation and has a given type. The types described by the model are grouped by the abstract class DataType. DataType subclasses are PrimitiveDataType, CollectionType, and ModellingConcept. PrimiteDataType includes Integer, Float, String and Boolean types. Collection describes a list of elements of a particular given type that are ordered, have no duplicates and are parameterized with an element type. ModellingConcept is imported from Domain Package and enables specifying arguments that explicit the type of an expected object version to be added during the execution of an add primitive.

As regards VariableAssignment, it denotes the mapping between a Variable and a RunTimeValue. This interface is not defined to specify operations. It is included to 
represent the run time values during the execution of an operation. RunTimeValue can be realized by different values like literal, object version, modelling concept, or AttribValue (value of an attribute of an object version, Fig. 2), depending on the variable type.

\subsection{Products of SADP}

In order to capture the versions generated during a SADP, the PVAM must be extended according to the particular design objects produced by that process. To this purpose, the Domain Package shown in Fig. 1 must be extended with concepts of the SADP domain. The products that constitute the design object types are taken from the Attribute-Driven Design Method (ADD) proposed in [4], and the architectural description language ACME [2]. The class diagram shown in Fig. 3 introduces these concepts and their relationships. This model is implemented by the instantiation of the classes of Domain package (Fig. 1). The classes presented in Fig. 3 are going to be instances of ModellingConcept and their properties are going to be instances of Attribute. Finally, the relationships of Fig. 3 will be instantiated from DomainRelationship in Domain package.

The ADD method is based on a recursive decomposition process where architectural patterns (or styles) are chosen at each stage to fulfil a set of quality scenarios. Then, component and connector types provided by architectural patterns are instantiated and functionality is allocated to them. The input to ADD is a set of requirements (functional and quality requirements). The quality requirements are expressed as a set of system specific quality scenarios, and the functional requirements are translated into a set of responsibilities [4]. Quality scenarios and responsibilities can be delegated to other components when the original component is refined. When the method iteration is finished, the designer verifies scenarios and sets an assessment.

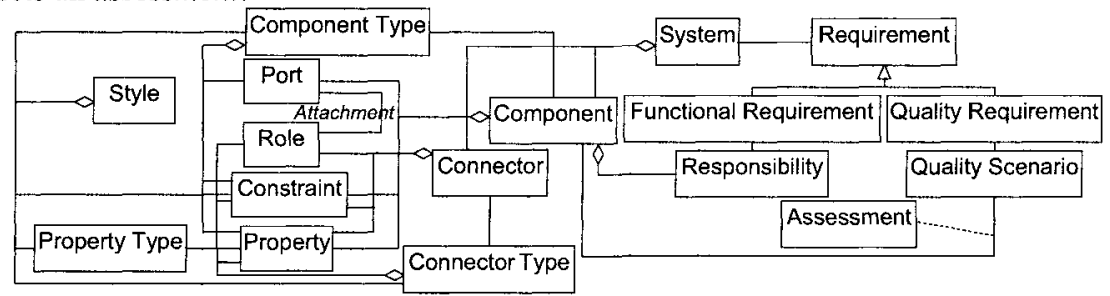

Fig. 3. Domain model for architecture based design

In $\mathrm{ADD}$, the different model versions are represented using various types of views. Only the component view is considered within the scope of this work in order to describe the architecture. Accordingly, ACME [2] has been chosen as the architectural description language. ACME defines a component as a computational element and data store of a system. A component may have multiple interfaces, each of which is termed port. The connectors represent interactions among components and have interfaces that are defined by a pair of roles. The systems comprise components and connectors, establishing attachments between roles and ports. In 
Fig. 3, the attachment concept is not considered as a modelling concept but as a relationship. Moreover, ACME proposes elements to document extra-structural properties of a system's architecture, as Properties. Furthermore, it is possible to attach constraints to design elements. With the aim of providing a more powerful language, ACME defines component, connector, and property type building blocks. On the basis of these modelling concepts, it is possible to define Families or Styles. They are defined by a set of property, component, and connector types and a set of constraints.

\subsection{Architectural Operations Specification}

As it was outlined in Section 3.1, PVAM must be extended in terms of the suitable operations for the SADP domain, like the ones listed in Table 1.

Fig. 4 presents functional specifications for some of the basic operations defined in Table 1. The other operations are defined in a similar way, but they are not shown due to lack of space. As seen in Fig. 4 , the operation addComponent $\left(s, c, l_{\text {Resps }}, l_{\text {Ports }}\right)$ is carried out by a series of operations. First, a version of component $c$ is added $(a d d(c))$. After that, a set of responsibilities (specified by list $l_{\text {Resp }}$ ) and ports (detailed by list $l_{\text {Ports }}$ ) are inserted. These operations are carried out by the addResponsibility ( $c$, $r)$ and addPort $(c, p)$ operations. Finally, a relationship between the new component $c$ and an existing system $s$ is included. This last operation is performed by the add primitive operation $(\operatorname{add}(\operatorname{rel}(s, c)))$. These operation specifications are implemented as instances of the Operation model introduced in Fig. 2.

In the same way as for basic operation, it is possible to define the special operations. Fig. 5 presents some examples. A function with a '?' symbol at the end indicates that it is interactive; thus, the user is asked about how to proceed. The interactive commands can be implemented as a special case of VarAssignment command (Fig. 2).

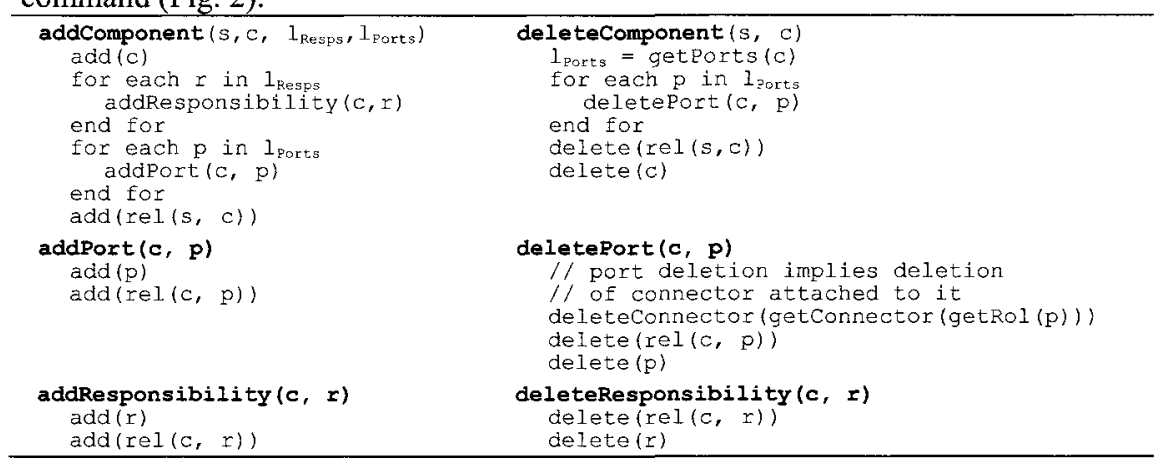

Fig. 4. Specifications of basic operations

The delegateResponsibility $\left(c_{1}, c_{2}\right)$ operation enables delegating a responsibility of component $c_{1}$ to component $c_{2}$. Thus, if a given responsibility is assigned to a component $c_{1}$ in a model version $m$ and a delegateResponsibility $\left(c_{1}, c_{2}\right)$ operation is included in the sequence of operations applied to $m$, then the resulting model version 
shows that the responsibilities delegated to $c_{2}$ will not be assigned to $c_{l}$. In a similar way, the operation delegateScenario proceeds.

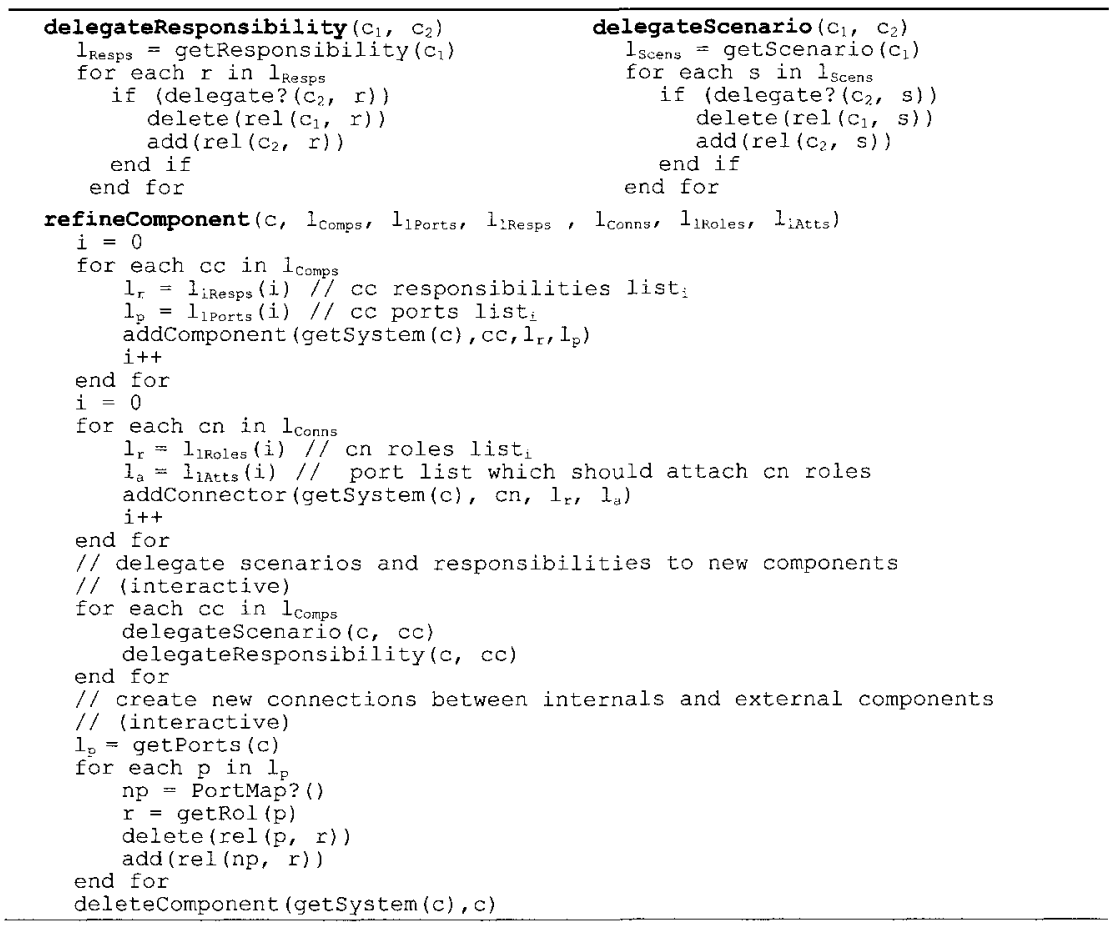

Fig. 5. Specifications of special operations

The refineComponent $\left(c, l_{\text {Comps }}, l_{\text {lPorts }}, l_{\text {lResps }}, l_{\text {Conns }}, l_{\text {lRoles }}, l_{\text {lAtts }}\right)$ operation, another example of special operation (Fig. 5), decomposes a component $c$ into one or more components given by the list $l_{\text {comps }}$. The ports and responsibilities of the new components are given by the lists $l_{\text {IPorts }}$ and $l_{\text {lResps }}$, respectively. Furthermore, a set of connectors among the new components is added. These connectors are specified by $l_{\text {Conns }}$ whose roles are given by the list $l_{\text {lRoles }}$ and the attachments by the list $l_{\text {lAtts }}$.

The operations that apply an architecture style [12], or an architectural pattern [13], refine a preexistent component with a new set of components and connectors that are instantiated from an architectural style/pattern. They interact with the designer asking for the responsibilities and scenarios delegation, as well as connectors mapping between external components and refined components. An example of applyStyle operation is defined in Fig. 6. In this case, the applyControlLoop operation is specified. This style proceeds from the process control paradigm and defines the architecture to activate various monitoring policies when different events coming from sensors are produced [14]. The monitoring policies may in turn produce other events or actions in response to predefined 
situations. Note that this operation can be considered as a specialization of refine Component operation. The knowledge on how to proceed in the refinement of component $c$ is given by the control loop style. Therefore, a series of addComponent operations is performed. The addComponent(s, $\{$ Diagnosis, TDiagnosis $\left.\},\left[P_{l}, P_{6}\right]\right)$ operation indicates that a component and two ports must be created. The component is called Diagnosis, whose modelling concept is TDiagnosis, an instance of ComponentType (see Fig. 3), and the ports are denominated $P_{I}$ and $P_{6}$.

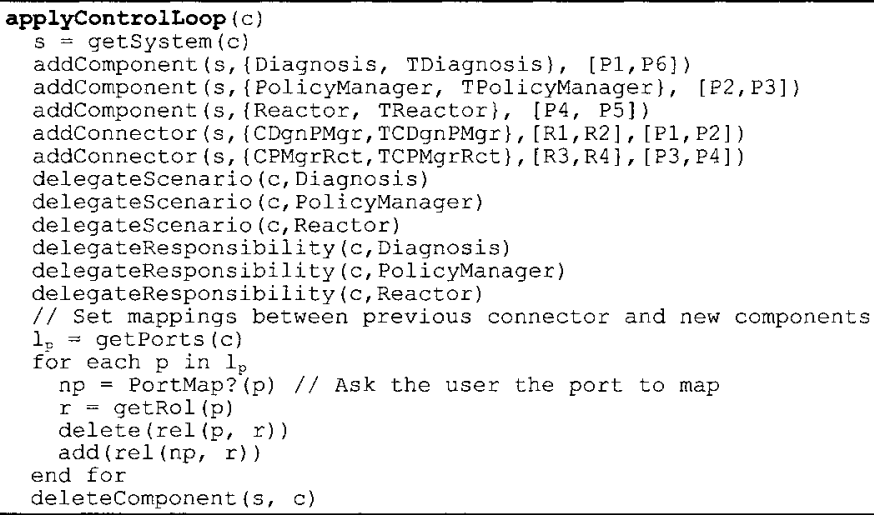

Fig. 6. Specification of applyControlLoop operation

\section{Case Study: Monitoring System for an Industrial Process}

The following case study describes the design of a monitoring system for an industrial process (see Fig. 7). It is based on classical case studies presented in other contributions [1, 4]. Monitoring activities are focused on the two core distillation columns: an extractive distillation column and a solvent stripping one, working together in a highly integrated manner. The system should monitor control loops and temperature sensors, by continued acquisition of real-time process data, tracking setpoint values, alarm conditions and outputs of valves, and comparing them with normal pattern behaviour. The system should also monitor process state, using realtime process data previously processed in combination with expert knowledge in order to maintain process stability and performance. Further functionalities are control flowrate sensors and validate material balances. In order to meet all these functional requirements, the system should be connected to input and output devices. Input devices allow the system to get the real time data from the process equipment and output devices are used by the system to inform the plant operator about process anomalies, like: solvent inventory buildup, sensor fault, abnormal process pattern, etc. The main functions considered in designing the monitoring system include: administration of users (process operator, plant supervisor, etc.) and permissions, 
configuration of input/output devices, priority-based event management, process diagnosis, specification of warning and process protective actions.

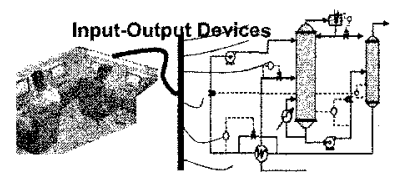

Fig. 7. Monitoring system for an industrial process

For reasons of space, only a sequence of operations of the model evolution is analyzed. Let us consider an intermediate model version $i$ (see Fig. 8) where the main components are: Control\&Diagnosis (with responsibilities in priority based event management, protective actions execution, warning launch, input/output devices configuration); UserInterface (with responsibilities related to user interaction issues: set parameters values, show information, rule administration); SensorActuatorLayer (with responsibilities like sending out commands to actuators, receiving information from sensors); and Configuration. From this model version, the designer chooses to refine the Control\&Diagnosis component by applying the applyControlLoop operation. This operation creates three new components: Diagnosis, PolicyManager, and Reactor. The applyControlLoop operation (see Fig. 6) asks the necessary information for delegating responsibilities, and for reconnecting previous connections to the new configuration.

Fig. 8 shows a partial view of the Version and Repository levels from which model version views can be inferred. This figure is focused on the version of Control\&Diagnosis evolution to a set of versions of components and connectors due to applyControlloop operation. A view of a model version is obtained from the knowledge in the Version and Repository levels. The object versions belonging to a model version are inferred by the belong $(v, m)$ predicate (Expression 3 ). Fig. 8 shows some object versions that belong to model version $i$ (Control\&Diagnosis ${ }_{v, 1}$, $P 1 C \& D_{v, l}, P 2 C \& D_{v, l}, P 3 C \& D_{v, 1}$ ). Given an object version (Control\&Diagnosis $v_{v, 1}$ ), it is possible to know its versionable object (Control\&Diagnosis ${ }_{o}$ ), which is linked with its design object type (modelling concept component, defined in Domain). All this information makes possible to reconstruct the elements of a model version view, as it is the Control\&Diagnosis component which is obtained from object version Control\&Diagnosis $_{v, 1}$ and versionable object Control\&Diagnosis. On the other hand, the expression 5 enables to retrieve the relationships among the object versions that belong to a given model version. Control\&Diagnosis ${ }_{o}$ has three ports named $P 1 C \& D_{o}, P 2 C \& D_{o}$, and $P 3 C \& D_{o}$ which have their respective object versions $P 1 C \& D_{v, 1}, P 2 C \& D_{v, 1}$, and $P 3 C \& D_{v, 1}$. Therefore, component Control\&Diagnosis $v_{v, 1}$ has ports $P 1 C \& D_{v, l}, P 2 C \& D_{v, 1}$, and $P 3 C \& D_{v, 1}$. 


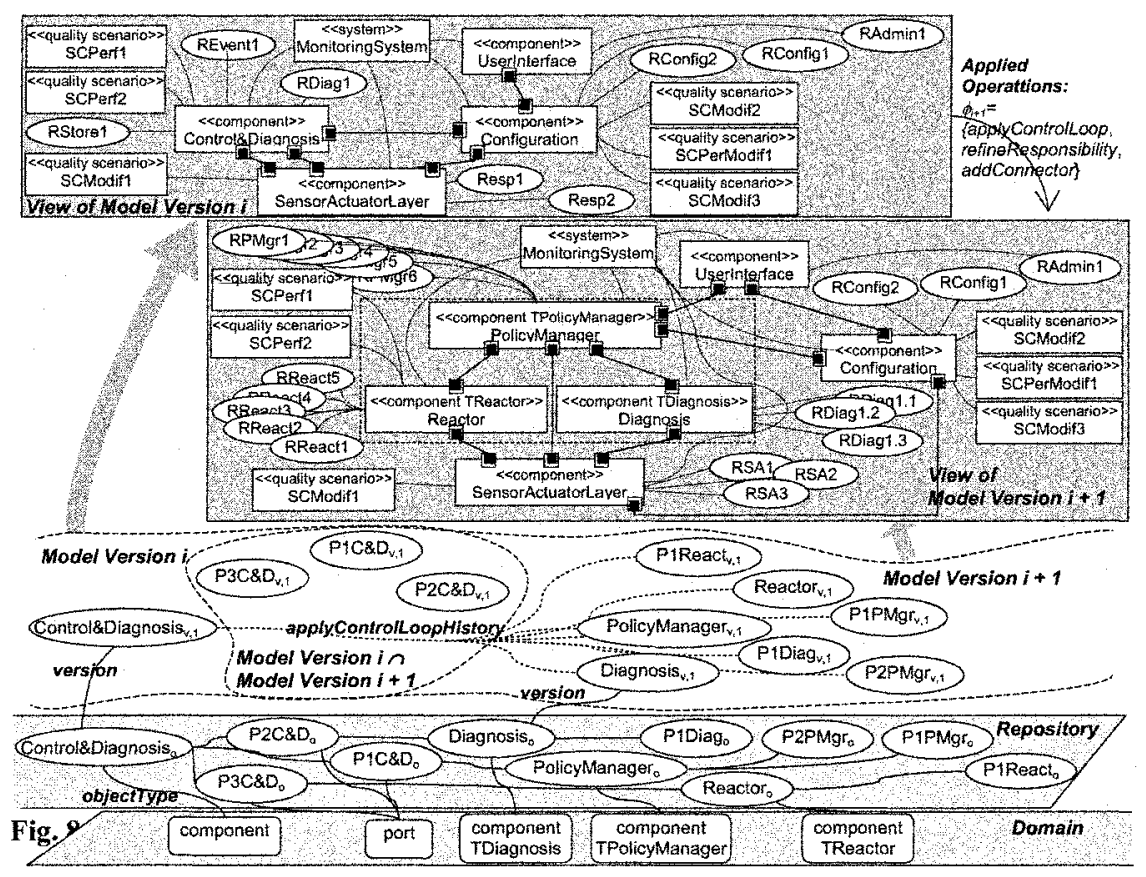

The applyControlLoop operation is applied on Control\&Diagnosis component (Control\&Diagnosis $v_{v, I}$ object version). This operation is traced by an instance of the history link (Fig. 1) which associates the previous object version (Control\&Diagnosis $_{v, 1}$ ) with the successor object versions (Reactor $_{v, l}$, Diagnosis Di, $_{v}$, PolicyManager $_{v, l}$, PIReact $_{v, 1}$, PIDiag $_{v, 1}, P 1 P M g r_{v, l}, P 2 P M g r_{v, l}$ in Fig. 8). PIC\&D $D_{v, 1}$, $P 2 C \& D_{v, l}$, and $P 3 C \& D_{v, l}$ object versions belong to both model version $i$ and model version $i+1$ because they were delegated from the original component (Control\&Diagnosis) to the newer ones by applyControlLoop operation.

Additionally, other operations were applied on model version $i$ to obtain model version $i+l$ that are not illustrated in Fig. 8 at version and repository levels. One of them arises due to the need of associating PolicyManager and Configuration components, so a new connection and their roles objects are added, applying addConnector operation. Using again operation addConnector, a new connection between PolicyManager and SensorActuatorLayer is added. It enables PolicyManager to receive information from, and send information to, SensorActuator (see Fig. 8, View of model version $i+1$ ).

It is important to note that the proposed extension of PVAM enables applied operations on SADP's products (Fig. 3) to be captured. For example, responsibilities are refined using refineResponsibility operation. The RDiag1 responsibility (Fig. 8, view of model version $i$ ) was refined on the following responsibilities: i) listening notifications of situations coming from SensorActuator (RDiag1.1); ii) getting 
devices information (RDiag1.2); iii) probing device (RDiagl.3) (Fig. 8, view of model version $i+1)$.

\subsection{Retrieving the History of Architectural Design Processes}

The model introduced allows tracing and recovering the history of the architectural design activities carried out by the designer during SADP. It is possible to ask about the history of model versions in terms of operation sequences that have generated a given model version, and also consult on the history of a particular object version, which allows to know how the evolution took place through the different versions. Fig. 9 shows an example of a history query to perform on the hypothetical monitoring system designed in current section. An actor would whish to know the sequence of operations that originated model version $i+1$ from the precedent model version $i$. The applied operations were applyControlLoop, refineResponsibility and addConnector, which can be seen in Fig. 8 of the case study. The resulting information allows knowing who carried out the operations, at what time and date, their arguments, the new elements incorporated to the design, the set of elements eliminated and what kind of modelling concepts they were. As shown in Fig. 9, additional information can be obtained, like the suboperations implied at the execution of the current one. Knowing which were the operations that gave rise to model version $i+1$ is useful for understanding the rationale associated with such a step because the architect knows the semantic of the operation and the intent.

\section{Conclusions}

The model proposed in this paper, an extension of PVAM, captures the operations that generate each design product during the SADP. Furthermore, it also offers an explicit mechanism to manage the different model versions generated during the SADP. Thus, it allows the tracing of the SADP and its resulting products, setting the grounds for learning and future reuse of the design process. This is a fundamental step towards the development of computational tools to support the SADP and to guide designers in the different activities of a design project. A related work [6] proposes a set of requirements which such tools should satisfy in order to adequately support the evolution of software architectures. The approach presented in this work meets a wide spectrum of those requirements: (i) First class architectural concepts, represented by the extensible domain model proposed; (ii) First class architectural design decisions, enabling specification of adequate operations for software architecture design representing design decisions made by the architect; (iii) Under-specification and incompleteness, allowed by the model evolution through discrete situations (model versions) increasing the level of abstraction; (iv) Explicit architectural changes, allowing capturing, managing and tracing of products of SADP, using explicit history links between different versions, which means that the operations applied through the design process are saved and, therefore, it is possible to reconstruct the history from an initial model version; (v) Support for modification, subtraction, and addition type changes, implemented by 
the primitive operations add, delete and modify. Those operations are also used in the definition of higher level operations representing more complex design operations like refining or styles application.

\begin{tabular}{|c|c|c|}
\hline $\begin{array}{l}\text { Model Version: Model } \\
\text { Precedent Model Versi } \\
\text { Applied Operations: }\end{array}$ & $\begin{array}{l}i+1 \\
\text { odel Version } \mathrm{i}\end{array}$ & \\
\hline $\begin{array}{l}\text { Operation applycontr } \\
\text { Model Version: Wodel } \\
\text { Arguments }\end{array}$ & on & Architect \\
\hline Argument Name & Value & Data Type \\
\hline Source Version & Control\&Diagnosis & Component \\
\hline Results, & (1) & -3 \\
\hline Object Version & Modelling Concept & Date Time \\
\hline PolicyManager & Component TPolicyManager & $01-06-200610: 56$ \\
\hline Reactor $_{\mathrm{v}, 1}$ & Component TPolicyManager & $01-06-200610.56$ \\
\hline$\ldots \ldots$ & 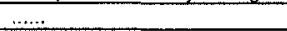 & $\ldots \ldots$ \\
\hline $\operatorname{RPMgr}_{v, 1}$ & Responsibility & $01-06-200610.56$ \\
\hline Deleted versions. & ५० & किए। \\
\hline Object Version & Modelling Concept & Date Time \\
\hline Control\&Diagnosis ${ }_{v 1}$ & Component & $01-06-200610: 56$ \\
\hline Rel C\&D Diag $n$ & Relation & $01-06-200610: 56$ \\
\hline Suboperations & किया & $10 \mathrm{mP}$ \\
\hline$(+)$ delegateRespons & & \\
\hline (+) delegateRespons & & \\
\hline$\sqrt{2}$ & & \\
\hline $\begin{array}{l}\text { Operation refineRespe } \\
\text { Model Version } \\
(+)\end{array}$ & $\mathrm{nl}$ & Arenitedt \\
\hline $\begin{array}{l}\text { Operation addConnec } \\
\text { Model Version Mode } \\
(+)\end{array}$ & Ac & Archisedt \\
\hline
\end{tabular}

Fig. 9. Partial view of the sequence of operations applied to model version $i$

Situation calculus, the formal background of the framework, allows us to represent the activities carried out during a SADP, and therefore, it enables the designer to get a better understanding of the information on how the various design objects (systems, components, connectors, functional requirements, quality requirements, quality scenarios, assessment, etc.) have been obtained. Thus, the history of operations performed on versions of design objects can be kept. Besides, this conceptual framework also provides the foundations for the proposal of formal means for detecting potential conflicts.

The framework could incorporate extensions to the Domain package, integrated to the version administration model, defining other characteristics not included by ADD or ACME. Furthermore, it uses an operational perspective where design decisions can be modelled by means of design operations. This approach is employed in other contributions [1,4]. The structure of the conceptual framework allows the easy definition of specific design operations, like applyControlLoop, by instantiating the Operation model (Fig. 2). This extension is possible without modifying the successor state axiom (Expression 3). 


\section{References}

1. A. Díaz Pace, A Planning-Based approach for the exploration of Quality-Driven design alternatives in Software Architectures, Tesis Doctoral (UNICEN, 2004).

2. D. Garlan, R. T. Monroe, D. Wile, Acme: Architectural Description of Component-Based Systems. Foundations of Component-Based Systems, edited by G.T. Leavens and M. Sitaraman (Cambridge University Press, 2000), pp. 47-68.

3. N. Medvidovic, D. Rosenblum, D. Redmiles, J. Robbins, Modeling Software Architectures in the Unified Modeling Language, ACM Transaction on Software Engineering and Methodology, 11(1), 2-57 (2002).

4. L. Bass, P. Clements, R. Kazman, Software Architecture in Practice: Second Edition (Addison-Wesley, 2003).

5. F. Bachmann, L. Bass, M. Klein, Preliminary Design of ArchE: A Software Architecture Design Assistant, Carnegie Mellon University, Technical Report CMU/SEI-2003-TR-021, 2003.

6. A. Jansen, J. Bosch, Evaluation of Tool Support for Architectural Evolution, in: Proceedings of the 19th IEEE International Conference on Automated Software Engineering (2004), pp. 375-378.

7. A. Tang, J. Han, Architecture Rationalization: A Methodology for Architecture Verifiability, Traceability and Completeness, in: 12th IEEE International Conference and Workshops on the Engineering of Computer-Based Systems (2005), pp. 135-144.

8. S. Gonnet, Un modelo integrado para la captura y administración del proceso de diseño, Tesis Doctoral (UNL, 2003).

9. R. Reiter, Knowledge in Action: Logical Foundation for Describing and Implementing Dynamical Systems (The MIT Press, 2001).

10. Object Management Group, OCL 2.0 Specification (2005), 2005-06-06.

11.E. Gamma, R. Helm, R. Johnson, K. Vlissides, Design Patterns. Elements of Reusable Object-Oriented Software (Addison-Wesley, 1995).

12.M. Shaw, D. Garlan, Software Architecture, Perspectives on an Emerging Discipline (Prentice-Hall, 1996).

13. F. Buschmann, R. Meunier, H. Rohnert, P. Sommerlad, M. Stal, Pattern-Oriented Software Architecture. A System of Patterns (John Wiley \& Sons, 1996).

14.M. Shaw, Beyond Objects: A Software Design Paradigm Based on Process Control, Carnegie Mellon University, Technical Report CMU-CS-94-154, 1994.

\section{Acknowledgments}

The authors wish to acknowledge the financial support received from CONICET, Universidad Tecnológica Nacional and Agencia Nacional de Promoción Científica y Tecnológica (PICT 12628). 